\title{
Effect of Gas Area Fraction and Reynolds Number on Mixing Flow in Microchannel having Superhydrophobic Transverse Grooves
}

\author{
Sak Jie Tan ${ }^{1}$, Kok Hwa Yu ${ }^{1,}{ }^{*}$, Eng Sam Yap ${ }^{1}$, Yew Heng Teoh ${ }^{1}$, Mohd Azmi Ismail ${ }^{1}$ \\ 1 School of Mechanical Engineering, Engineering Campus, Universiti Sains Malaysia, 14300 Penang, Malaysia
}

ARTICLE INFO ABSTRACT

\section{Article history:}

Received 19 April 2020

Received in revised form 17 June 2020

Accepted 23 June 2020

Available online 29 June 2020

\section{Keywords:}

water repellent; micromixer; surface roughness; air cavity

\begin{abstract}
Mixing in microchannel cannot be typically carried out by forcing liquid streams in turbulent flow to enhance its mixing performance. The Reynolds number (Re) of mixing flow is typically below the critical value where transition to turbulence would occurs. It is thus a challenging problem for micromixers to mix fluids of different species within a desired range of mixing channel length in the absence of enhanced mixing techniques. This study presents numerical approach that investigates the mixing performance in microchannel having alternating superhydrophobic grooves and ribs. A channel that bounded by two parallel walls patterned with alternating superhydrophobic grooves and ribs is employed throughout the study and the simulations show that normalized mixing length increases monotonically with the increase of gas area fraction. The mixing performance is also explored in the bulk region and region close to the superhydrophobic walls, at $y / H=0.1,0.5$ and 0.9 . It is found that the presence of superhydrophobic wall tends to give rise to a longer mixing length. Besides that, the influence of flows Reynolds number is also examined and it show that the mixing length is relatively similar as compared to that of smooth wall at low Reynolds numbers. This is mainly due to the use of small textured features, i.e., superhydrophobic grooves of $\Lambda=0.1$. With the use of superhydrophobic wall, it could be potentially beneficial in mixing channel with limited pumping power without significantly affect the mixing performance.
\end{abstract}

\section{Introduction}

For micromixers, rapid mixing is crucial in Lab-on-a Chip and MEMS applications [1-3]. In order to further improve the mixing performance, it is important to understand the nature of fluid mixing for internal flow. Microchannel is defined as narrow channel with dimensions ranging between $1 \mu \mathrm{m}$ and $1 \mathrm{~mm}$. Beyond $1 \mathrm{~mm}$, the fluid flow behaves similar to that of macroscopic flow. In microchannel, mixing of fluids basically occurs at low Reynolds number due to low flow rate, arising from the low

\footnotetext{
* Corresponding author.

E-mail address: yukokhwa@usm.my (Kok Hwa Yu)
} 
pumping power available. Therefore, the fluid flow in the channel is mostly restricted to laminar flow and it requires mixing techniques to improve the mixing performance. Mixing flow rates are manipulated by molecular diffusion at low Reynolds number, mixing length $\left(L_{\text {mix }}\right)$ and residence time could be longer in the absence of enhanced mixing technique [4]. There are several existing mixing techniques that were recommended and investigated previously. These techniques can be classified as either active technique or passive technique. For active mixing, it can be accomplished by microstirrers [5], acoustic mixing [6], periodic fluid pulsation [7], thermal mixing enhancement [8], electrokinetic mixing enhancement [9], etc. Although the active techniques are mainly user controllable methods, these techniques often require external power source to operate and it is extremely strenuous to fabricate and harder to integrate into the microfluidics device for mixing purposes. However, the passive mixing techniques do not require additional external mechanism to drive the mixing process. It relies largely on hydrodynamics of the flow [10], alteration of the surface topology [11] and changes in the channel geometry [12]. Apart from mixing, alteration of surface topology using groove-rib can be pursued in enhancing heat transfer [13-14]. This surface feature consists of a series of ridges patterned on the channel wall. A recent study of fabrication of polydimethylsiloxane (PDMS) microfluidic devices is introduced by Gobbi et al., [15] using soft lithography methods and plasma-based bonding. In addition, different microchannel configuration like triangular cavities microchannel [16] can be pursued for mixing flow.

For water repellent surface, when a liquid droplet is placed on solid surface, it could retain the form of a droplet or spread out on the surface to form a thin liquid film [17-18]. The advantage of water repellent surface is the ability in minimizing the flow resistance in order to gain higher mass flow rate in narrow medium such as microtube when the external power source is restricted [19-21]. Recently, this water repellent surface is studied and employed in various practical applications such as lab on chip technology [22], thermal management [23], condensation [24] and etc. This physiochemical feature is represented as surface wettability and this surface feature are important in many different fields such as biology, painting of cars and microsystems engineering [25].

The interest to create artificial superhydrophobic surface was enlightened from the diverse superhydrophobic surface found in nature such as water-repellent properties of lotus leaf, insect legs and butterfly wings. Recent synthetic surfaces have been developed that are capable of obtaining contact angles that can reach $\theta=180^{\circ}$ [26]. High water-repelling property can be achieved when surface is both chemically hydrophobic and physically rough. Superhydrophobic surface can cause water or oils to roll off leaving little or no residue, and carry away any resting surface contamination [27]. To mimic the effects of superhydrophobic surface, it can be represented either by imposing an effective velocity slip length [28] or by having a mixed boundary condition along superhydrophobic wall. Under laminar flow conditions, rapid mixing is not easily achieved. Recently, apparent slip effects of water flows in hydrophilic and hydrophobic microchannels was examined by Choi et al., [29]. The work of Choi et al., [29] shows that there exists a discernable difference between the boundary condition between water at a hydrophilic and at a hydrophobic surface. The slip length is indicated to increase approximately linearly with shear rate over hydrophobic surface whereas the existence of slip over the hydrophilic surface remains uncertain due to sensitivity of analysis with nanometer uncertainties in channel height. Besides that, research of flow and mixing in channel with misaligned superhydrophobic walls was studied by Nizkaya et al., [30] to analyze the mixing properties of superhydrophobic channels with arbitrary oriented textures. They showed that the transverse shear leads to complex flow patterns which lead to new mechanism of passive mixing at low Reynolds number.

However, despite there were some existing studies on mixing flow in channel employing superhydrophobic surfaces, the influence on mixing length in the superhydrophobic surface is still 
largely unknown. In this paper, superhydrophobic surface with rib-groove periodic structures is employed in microchannel and the effects of relevant factors, i.e., dimensionless gas area fraction and flow Reynolds number, on mixing length are investigated.

\section{Methodology}

Consider fluid flow in a channel that is bounded by two parallel walls patterned with alternating superhydrophobic grooves and ribs arranged transversely with the flow direction, as illustrated in Figure 1. Both of the walls are separated by a distance $H$ and it is used as the characteristic length for this study. The $x-, y-$ and $z$-direction represents the axial, vertical and horizontal direction, respectively. There are two different flow inlets, i.e., Inlet $A$ and Inlet $B$, with equal height that contain Fluid $A$ and Fluid $B$, respectively.

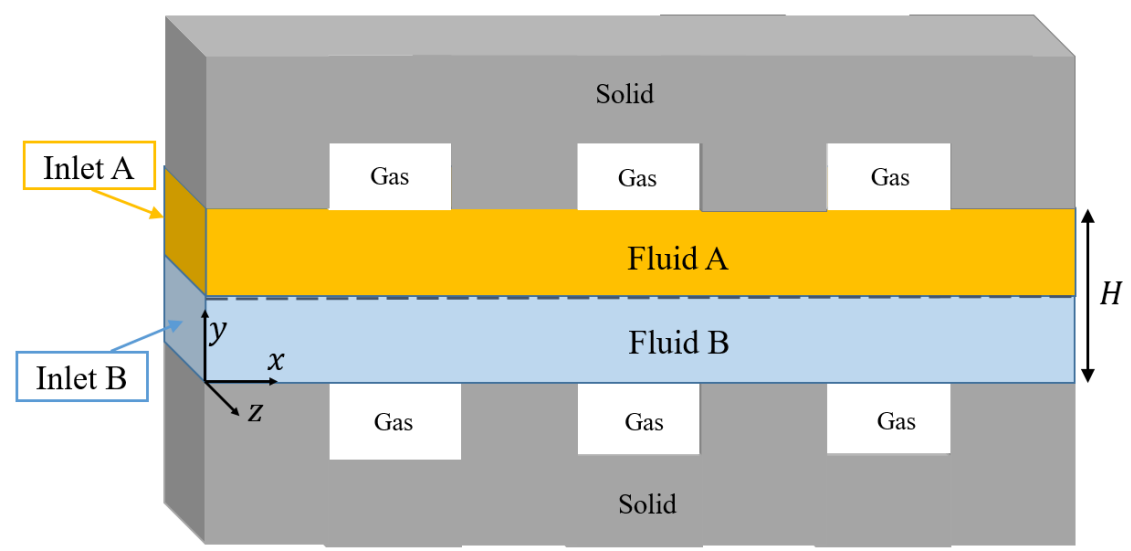

Fig. 1. Schematic diagram depicting flow in a plane channel having superhydrophobic transverse grooves and ribs

Under the hydrophobic condition, the gas is assumed to be trapped inside the grooves and this give rise to liquid-gas interface over the grooves. In this study, the liquid-gas interface is assumed to be perfectly flat. The groove-rib unit length is represented by $E$ while the width of groove is denoted by $e$, as illustrated in Figure 2. In this study, the effects of the size of surface microstructures are explored via the dimensionless gas area fraction $\delta=e / E$ and normalized grooves-rib periodic spacing $\Lambda=E / H$.

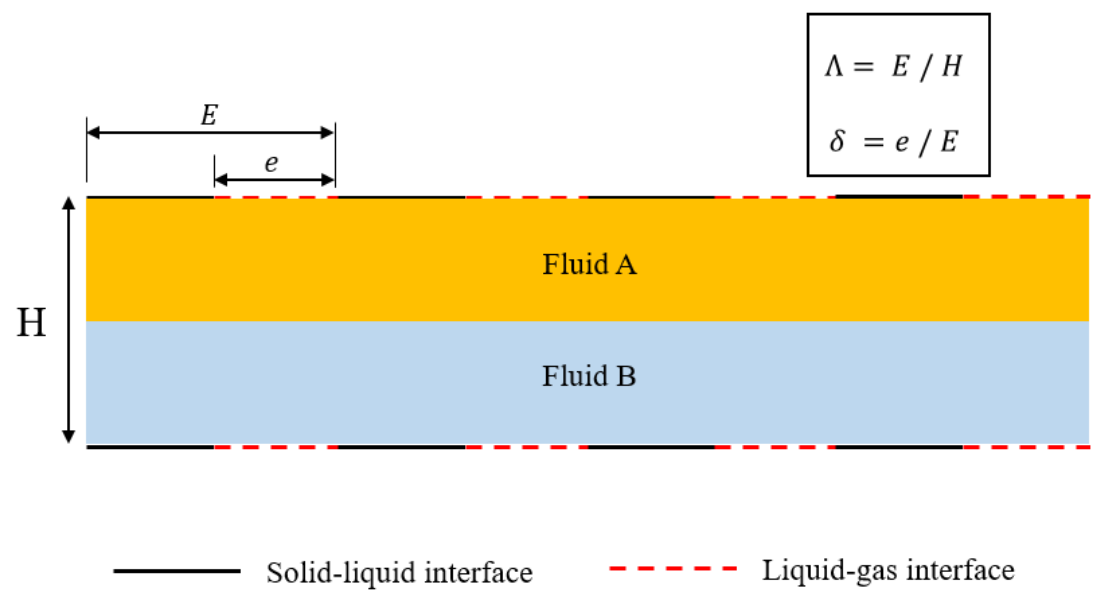

Fig. 2. Schematic of geometry of superhydrophobic transverse grooves in mixing channel 
The mixing liquid flow problem can be simulated by solving the fluid flow problem coupled with an advection-diffusion equation for species concentration. In this study, flow of an incompressible Newtonian fluid is considered. Since the liquid mixing problem doesn't involve any chemical reaction, thus it is neglected. The governing equations for this study are thus stated as follow:

$\nabla \cdot \mathbf{u}=0$

$\rho(\nabla \mathbf{u}) \mathbf{u}=-\nabla P+\mu \nabla^{2} \mathbf{u}$

u. $(\nabla c)=D \nabla^{2} c$

where $\rho$ is the density of the fluid, $\mu$ is the dynamic viscosity, $P$ is the pressure field, and $\mathbf{u}$ is the velocity field. In Eq. (3), $c$ is the species concentration and $D$ is the diffusivity. No-slip condition is implemented along the solid-liquid interface (ribs) which will give rise to zero fluid velocity over the solid surface. To mimic the vanishing shear stress over the liquid-gas interface, the shear-free condition is thus applied along the liquid-gas interface. At both inlets (i.e., Inlet A and Inlet B), uniform velocity based on the flow Reynolds number $\left(R e=\rho U_{a v g} H / \mu\right)$ is applied where $U_{\text {avg }}$ is the flow average velocity.

The pressure-velocity coupling is solved via SIMPLE scheme. The numerical solution for pressure and momentum are based on second-order and second-order upwind schemes, respectively. The convergence criteria with scaled residuals of $10^{-10}$ for continuity, momentum and species transport equations are employed in this study. The governing equations (i.e., Eqs. (1) - (3)) along with the boundary conditions mentioned are thus solved using ANSYS FLUENT 18.1, a finite volume based computational fluid dynamics (CFD) software package. The species concentration over the flow domain is thus obtained to determine the mixing length. The mixing length $L_{\text {mix }}$ is calculated based on the length required along the centerline to achieve $1 \%$ difference variation in species concentration as compared to its fully mixed mole fraction.

\section{Results and Discussion}

Before the scaling effects of the superhydrophobic microstructures were explored, grid independent test is first performed. It is assumed that Fluid $A$ (flowing through Inlet $A$ ) has relative concentration $c^{*}=c / c_{0}$ of 1 and Fluid $\mathrm{B}$ (flowing through Inlet $\mathrm{B}$ ) has $c^{*}=0$. The average concentration of a perfect mixture is indicated by $c_{0}$. In this study, constant value for relative density between Fluid A and Fluid B (i. e., $\rho_{A} / \rho_{B}=2$ ) is employed. For grid independent test, simulations on mixing flow with Reynolds number of $R e=1$ and Schmidt number $S c=100$ were carried out using four different grid resolutions, i.e., $N_{X} \times N_{y}=400 \times 20,800 \times 40,1600 \times 80$ and $3200 \times$ 160. $N_{X}$ and $N_{y}$ are the number of elements in the axial direction and vertical direction, respectively. The elements used are uniformly spaced along $x-$ and $y$ - direction. Both upper and lower walls patterned transverse superhydrophobic grooves of $\Lambda=0.1$ and $\delta=0.5$ is employed. It is worth to mention that in this study, a sufficiently long channel length, i.e., $20 \mathrm{H}$, is used to yield the mixing length.

The relative concentration distribution along the centreline (i.e., $y / H=0.5$ ) is illustrated in Figure 3. As can be observed from this figure, the relative concentration rises until it become a perfect mixture with $c^{*}=0.5$. It is worth to note that the relative concentration distribution for simulations using $N_{X} \times N_{y}=1600 \times 80$ and $N_{X} \times N_{y}=3200 \times 160$ are almost identical. Using the relative concentration distribution, the normalized mixing length $\left(L_{\text {mix }} / H\right)$ can be calculated as indicated by 
the red dashed line in Figure 3 . The normalized mixing length $L_{\text {mix }} / H$ yielded by different grid resolutions used are illustrated in Figure 4. As can be observed in Figure 4, simulation with grid solution of $N_{X} \times N_{y}=1600 \times 80$ yields normalized mixing length of 0.326 . Doubling the number of elements in both the axial and vertical direction to $N_{X} \times N_{y}=3200 \times 160$ would only results in a variation of less than $1.7 \%$ for $L_{m i x} / H$ yielded. Therefore, the grid solution $\left(N_{X} \times N_{y}=1600 \times 80\right)$ is best employed.

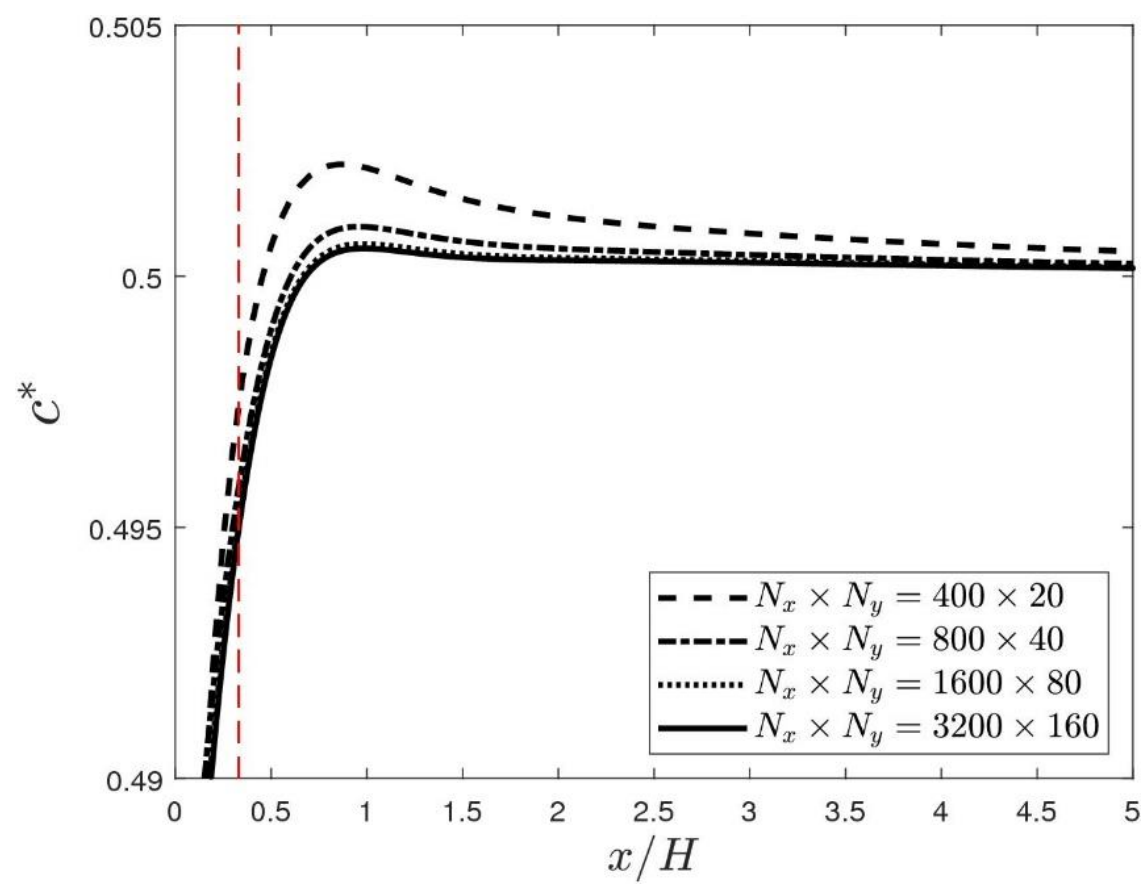

Fig. 3. Relative concentration $c^{*}$ distribution along the centerline for mixing channel having superhydrophobic transverse grooves of $\Lambda=$ 0.1 and $\delta=0.5$ using different grid resolutions

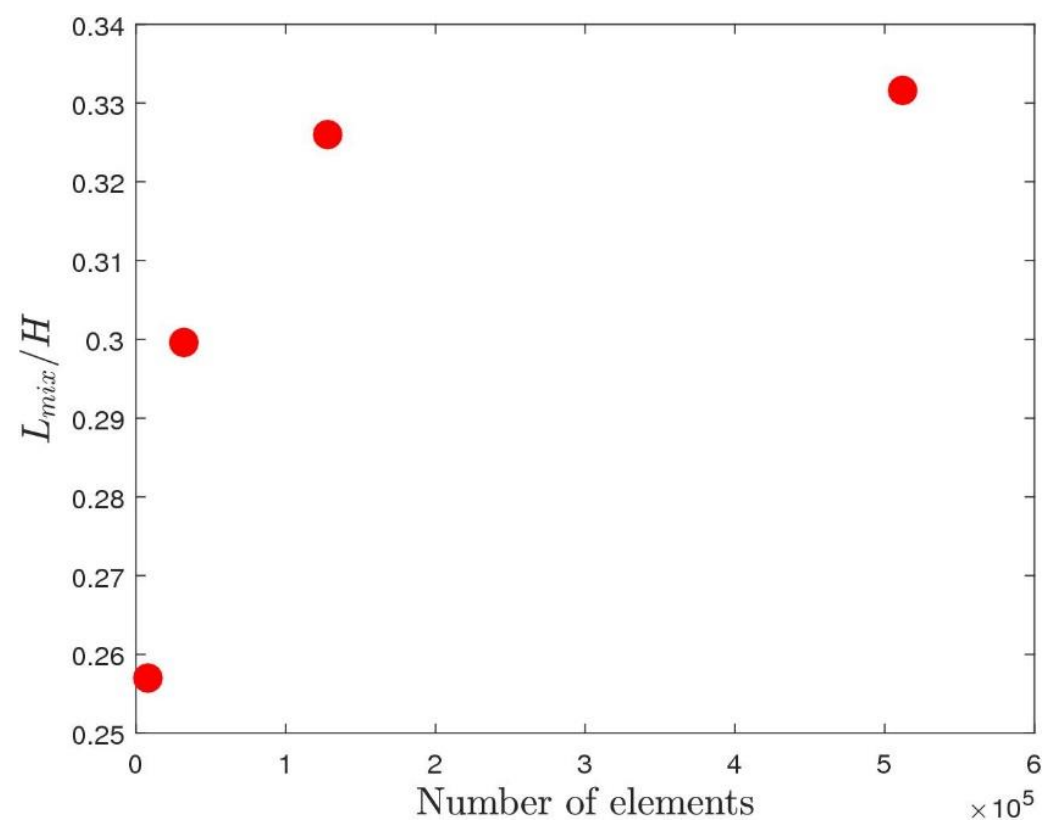

Fig. 4. Normalized mixing length $L_{\text {mix }} / H$ for mixing flow over superhydrophobic transverse grooves of $\Lambda=0.1$ and $\delta=0.5$ using different grid resolutions 


\subsection{Effect of Dimensionless Gas Area Fraction on Mixing Length}

To explore the effects of dimensionless gas area fraction on mixing flow in plane channel, three different gas area fractions, i.e. $\delta=0.25,0.5$ and 0.75 were employed. Gas area fraction represents the ratio of area occupied by liquid-gas interface over a groove-rib period. Relatively, a smaller value of $\delta$ indicates that the area of liquid-gas interface is smaller and vice versa. For $\delta=0.5$, the area occupied by liquid-gas interface and solid-liquid interface are equal. As can be observed in Figure 5, at $R e=1$ with superhydrophobic transverse grooves of $\Lambda=0.1$, employing this superhydrophobic wall would yield a longer mixing length. The increase in normalized mixing length is monotonic with the increase of gas area fraction. At $\delta=0$, the normalized mixing length is 0.3134 . Employing gas area fraction of $0.25,0.5$ and 0.75 will give rise to $1.98 \%, 4.7 \%$ and $11.77 \%$ increase in the normalized mixing length. This indicates that having this salient surface will yield a higher required length along the centerline for fluid to reach perfectly mixed fluid, as indicated in Figure 6(b).

The mixing performance is also explored in the region close to the superhydrophobic walls, at $y / H=0.1$ as depicted in Figure $6(\mathrm{a})$ and at $y / H=0.9$ as depicted in Figure $6(\mathrm{c})$. At $y / H=0.1, c^{*}$ tends to increase, starting with zero as prescribed at the inlet. At $y / H=0.9$, the trend is vice versa. With superhydrophobic wall, it tends to give rise to a relatively slower mixing. This can be due to the increase in the fluid flow close to the wall as it allows more fluid flow in that region, especially when a larger value of $\delta$ is employed. However, the increase in the velocity magnitude is dominantly in the axial direction. With the increase in the initial force in the bulk of the flow and a delaying effect is caused to exhibits a longer mixing length for fluid to achieve complete mixing.

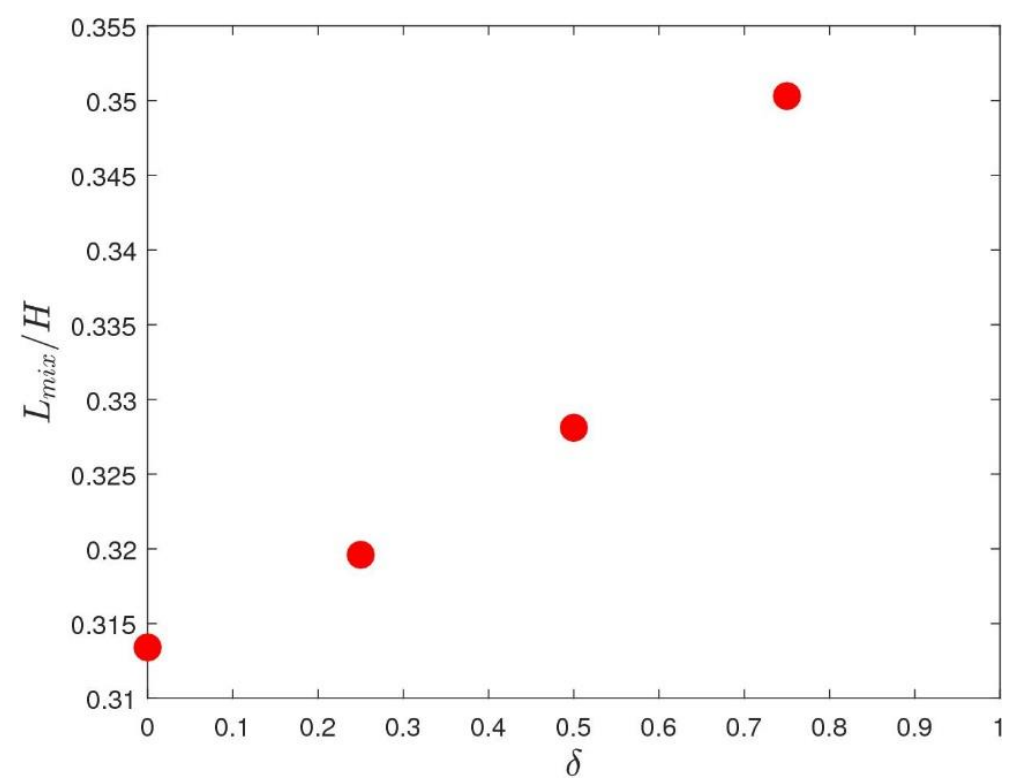

Fig. 5. Normalized mixing length $L_{\text {mix }} / H$ for mixing flow over superhydrophobic transverse grooves of $\Lambda=0.1$ at $\operatorname{Re}=1$ 
(a)

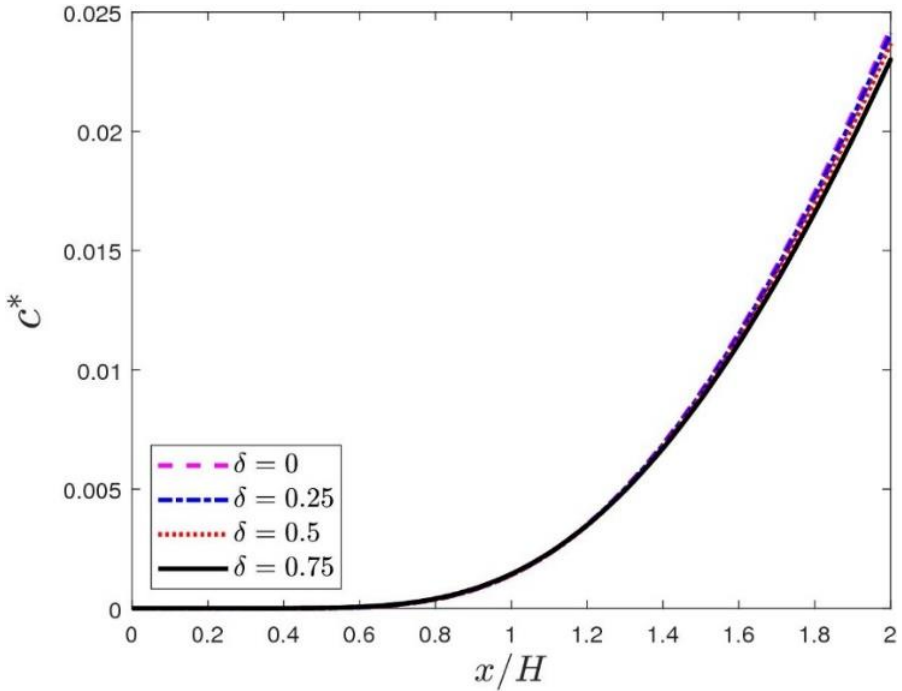

(b)

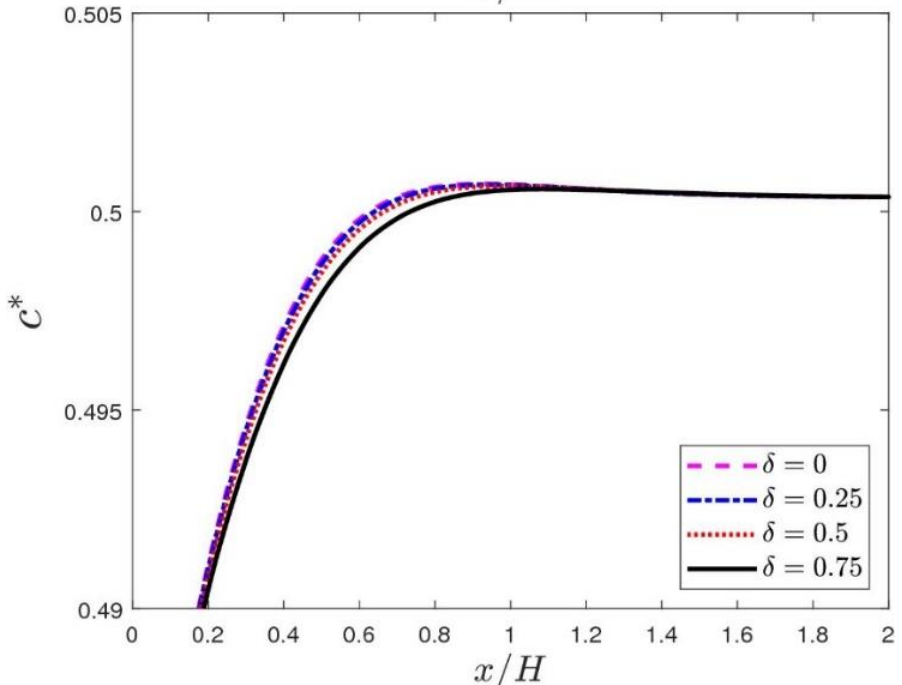

(c)

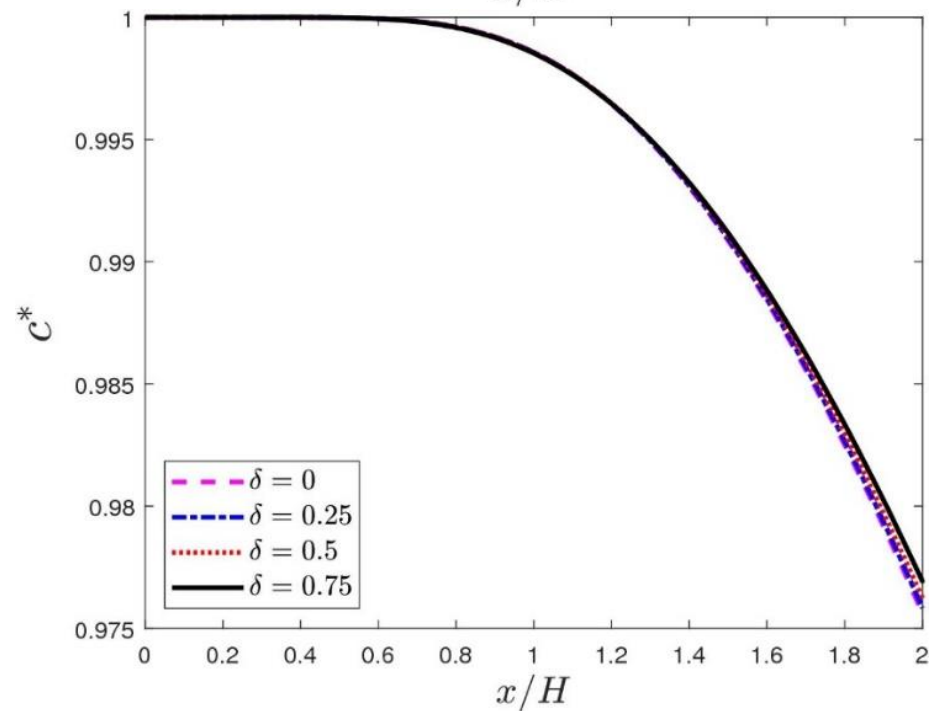

Fig. 6. Relative concentration $c^{*}$ distribution in mixing flow over superhydrophobic transverse grooves of $\Lambda=0.1$ at $R e=1$ for (a) $y / H=0.1$, (b) $y / H=0.5$ and (c) $y / H=0.9$ 


\subsection{Effect of Flow Reynolds Number}

Apart from investigating the influence of gas area fraction, the influence of flow Reynolds number is also explored. Employing superhydrophobic transverse grooves of $\Lambda=0.1$ and $\delta=0.5$, the fluid flow scenarios with Reynolds number ranging between $R e=1$ to $R e=50$ were simulated. As depicted in Figure 7, having higher Reynolds number (fluid flow), this will increase the normalized mixing length. The increasing trend is found to be consistent with the increase in the normalized mixing length for flow scenarios with smooth wall. For smooth wall, at $R e=50$, the mixing length is expected to be 4.66 times of the channel height. Having superhydrophobic grooves of $\Lambda=0.1$ and $\delta=0.5$, it will only give rise to additional mixing length of $1.8 \%$.

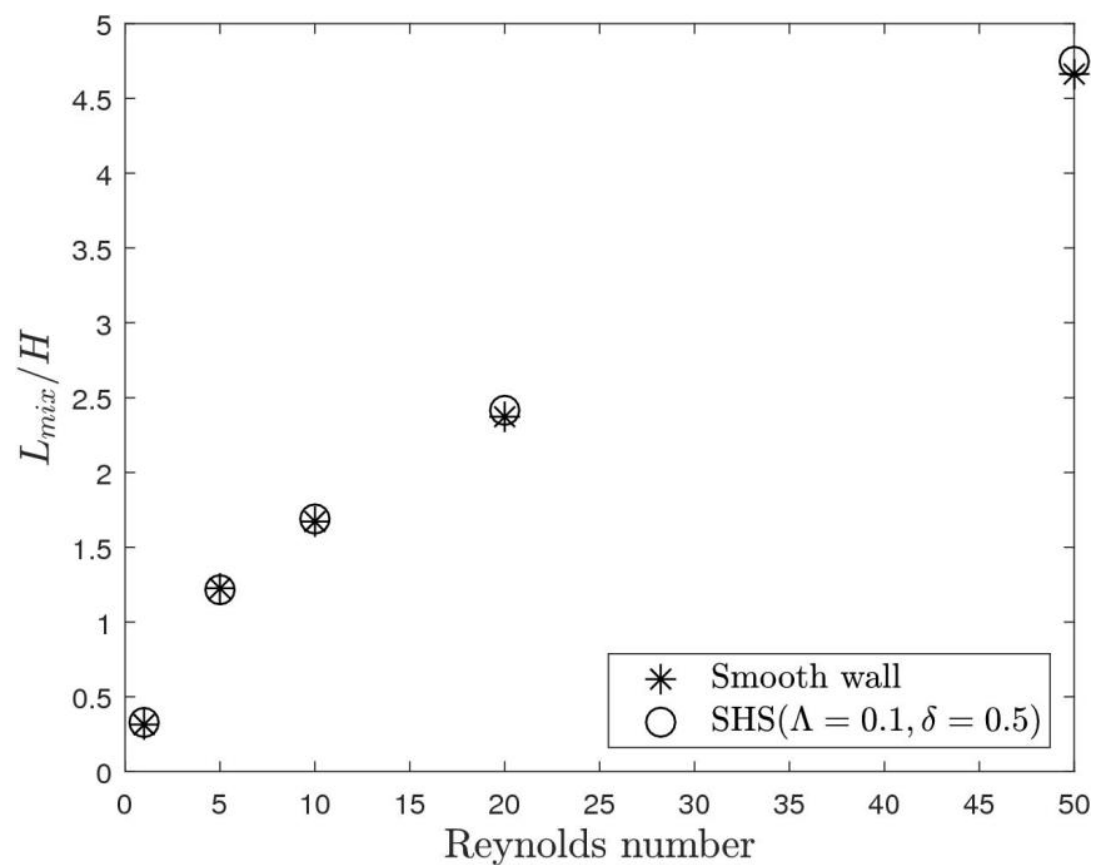

Fig. 7. Comparison on $L_{m i x} / H$ for mixing flow over smooth wall and superhydrophobic transverse grooves of $\Lambda=0.1$ and $\delta=0.5$ at different flow Reynolds number

At lower Reynolds numbers, the differences are deemed small and thus not significant. This is mainly arising from the use of superhydrophobic grooves of $\Lambda=0.1$. With this surface configuration, the size of the surface texture is relatively small as compared to the channel height, thus inducing a weaker flow enhancement over the bulk of the flow. In spite of the need for small additional mixing length, the use of superhydrophobic wall can be potentially beneficial in mixing channel with limited pumping power without significantly affect the mixing performance.

\section{Conclusions}

In this study, mixing flow in channel having superhydrophobic transverse grooves have been investigated. At low Reynold number, the laminar flow is mainly relied on molecular diffusion and can be influenced by implementing superhydrophobic surface. In this study, the effects of dimensionless gas area fraction on mixing length in microchannel at $R e=1$ with superhydrophobic transverse grooves of $\Lambda=0.1$ was studied with a series of numerical simulations. At $\delta=0$, the normalized mixing length is 0.3134 . Employing gas area fraction of $0.25,0.5$ and 0.75 will give rise to $1.98 \%, 4.7 \%$ and $11.77 \%$ increase in the normalized mixing length, respectively. It indicates that the 
normalized mixing length increases monotonically with the increase of gas area fraction. Apart from that, the effect of flow Reynolds number for superhydrophobic wall was investigated. The flow Reynolds number was simulated in the range between $R e=1$ to $R e=50$. It shows that mixing length is relatively similar as compared to that of smooth wall at low Reynolds numbers due to the use of superhydrophobic grooves of $\Lambda=0.1$. From this study, it indicates that superhydrophobic wall could be potentially beneficial in mixing channel with limited pumping power without significantly affect the mixing performance.

\section{Acknowledgement}

The authors acknowledge Universiti Sains Malaysia RUI Grant No.: 1001/PMEKANIK/8014147 and School of Mechanical Engineering for the financial support.

\section{References}

[1] van den Berg, Albert. "Labs on a chip for health care applications." In The 14th International Conference on Miniaturized Systems for Chemistry and Life Sciences. 2010.

[2] Streets, Aaron M., and Yanyi Huang. "Chip in a lab: Microfluidics for next generation life science research." Biomicrofluidics 7, no. 1 (2013): 011302.

https://doi.org/10.1063/1.4789751

[3] Wang, Hengzi, Pio lovenitti, Erol Harvey, and Syed Masood. "Optimizing layout of obstacles for enhanced mixing in microchannels." Smart materials and structures 11, no. 5 (2002): 662.

https://doi.org/10.1088/0964-1726/11/5/306

[4] Ou, Jia, Geoffrey R. Moss, and Jonathan P. Rothstein. "Enhanced mixing in laminar flows using ultrahydrophobic surfaces." Physical Review E 76, no. 1 (2007): 016304.

https://doi.org/10.1103/PhysRevE.76.016304

[5] Lu, Liang-Hsuan, Kee Suk Ryu, and Chang Liu. "A magnetic microstirrer and array for microfluidic mixing." Journal of microelectromechanical systems 11, no. 5 (2002): 462-469.

https://doi.org/10.1109/JMEMS.2002.802899

[6] Wang, S. S., Z. J. Jiao, X. Y. Huang, C. Yang, and N. T. Nguyen. "Acoustically induced bubbles in a microfluidic channel for mixing enhancement." Microfluidics and nanofluidics 6, no. 6 (2009): 847-852.

https://doi.org/10.1007/s10404-008-0357-6

[7] Goullet, Arnaud, lan Glasgow, and Nadine Aubry. "Dynamics of microfluidic mixing using time pulsing." In Conference Publications, vol. 2005, no. Special, p. 327. American Institute of Mathematical Sciences, 2005.

[8] Tsai, Jr-Hung, and Liwei Lin. "Active microfluidic mixer and gas bubble filter driven by thermal bubble micropump." Sensors and Actuators A: Physical 97 (2002): 665-671.

https://doi.org/10.1016/S0924-4247(02)00031-6

[9] Shin, S. M., I. S. Kang, and Yoon-Kyoung Cho. "Mixing enhancement by using electrokinetic instability under timeperiodic electric field." Journal of Micromechanics and Microengineering 15, no. 3 (2004): 455.

https://doi.org/10.1088/0960-1317/15/3/005

[10] Lee, Gwo-Bin, Chih-Chang Chang, Sung-Bin Huang, and Ruey-Jen Yang. "The hydrodynamic focusing effect inside rectangular microchannels." Journal of Micromechanics and Microengineering 16, no. 5 (2006): 1024. https://doi.org/10.1088/0960-1317/16/5/020

[11] Tserepi, Angeliki, Evangelos Gogolides, Katerina Tsougeni, Vassilios Constantoudis, and Evangelos S. Valamontes. "Tailoring the surface topography and wetting properties of oxygen-plasma treated polydimethylsiloxane." Journal of applied physics 98, no. 11 (2005): 113502.

https://doi.org/10.1063/1.2136421

[12] Yang, Jing-Tang, Ker-Jer Huang, and Yu-Chun Lin. "Geometric effects on fluid mixing in passive grooved micromixers." Lab on a Chip 5, no. 10 (2005): 1140-1147. https://doi.org/10.1039/b500972c

[13] Japar, Wan Mohd Arif Aziz, Nor Azwadi Che Sidik, and Shabudin Mat. "A comprehensive study on heat transfer enhancement in microchannel heat sink with secondary channel." International Communications in Heat and Mass Transfer 99 (2018): 62-81. https://doi.org/10.1016/j.icheatmasstransfer.2018.10.005 
[14] Japar, Wan Mohd Arif Aziz, Nor Azwadi Che Sidik, R. Saidur, Yutaka Asako, and Mohd Irwan Mohd. "Temperature Minimization on the Substrate of a Heat Sink By Rib-Groove Microchannel Heat Sink with Effective Energy Consumption: Groove Geometry Parameter Effects." CFD Letters 11, no. 9 (2019): 1-13.

[15] Lima, Renato S., Leandro Y. Shiroma, Maria HO Piazzetta, and Angelo L. Gobbi. Fabrication of PDMS microfluidic devices using soft lithography and plasma-based bonding. Technical memorandum 062014-CNPEMMicrofabrication laboratory, 2014.

[16] Beng, Soo Weng, and Wan Mohd Arif Aziz Japar. "Numerical analysis of heat and fluid flow in microchannel heat sink with triangular cavities." Journal of Advanced research in fluid mechanics and thermal sciences 34, no. 1 (2017): 1-8.

[17] Zorba, Vassilia, Emmanuel Stratakis, Marios Barberoglou, Emmanuel Spanakis, Panagiotis Tzanetakis, Spiros H. Anastasiadis, and Costas Fotakis. "Biomimetic artificial surfaces quantitatively reproduce the water repellency of a lotus leaf." Advanced materials 20, no. 21 (2008): 4049-4054. https://doi.org/10.1002/adma.200800651

[18] Callies, Mathilde, and David Quere. "On water repellency." Soft matter 1, no. 1 (2005): 55-61. https://doi.org/10.1039/b501657f

[19] Lauga, Eric, and Howard A. Stone. "Effective slip in pressure-driven Stokes flow." Journal of Fluid Mechanics 489 (2003): 55-77. https://doi.org/10.1017/S0022112003004695

[20] Maynes, D., B. W. Webb, and J. Davies. "Thermal transport in a microchannel exhibiting ultrahydrophobic microribs maintained at constant temperature." Journal of Heat Transfer 130, no. 2 (2008). https://doi.org/10.1115/1.2789715

[21] Ou, Jia, Blair Perot, and Jonathan P. Rothstein. "Laminar drag reduction in microchannels using ultrahydrophobic surfaces." Physics of fluids 16, no. 12 (2004): 4635-4643.

https://doi.org/10.1063/1.1812011

[22] Karatay, Elif, A. Sander Haase, Claas Willem Visser, Chao Sun, Detlef Lohse, Peichun Amy Tsai, and Rob GH Lammertink. "Control of slippage with tunable bubble mattresses." Proceedings of the National Academy of Sciences 110, no. 21 (2013): 8422-8426.

https://doi.org/10.1073/pnas.1304403110

[23] Enright, Ryan, Marc Hodes, Todd Salamon, and Yuri Muzychka. "Isoflux nusselt number and slip length formulae for superhydrophobic microchannels." Journal of Heat Transfer 136, no. 1 (2014).

https://doi.org/10.1115/1.4024837

[24] Enright, Ryan, Nenad Miljkovic, Ahmed Al-Obeidi, Carl V. Thompson, and Evelyn N. Wang. "Condensation on superhydrophobic surfaces: the role of local energy barriers and structure length scale." Langmuir 28, no. 40 (2012): 14424-14432.

https://doi.org/10.1021/la302599n

[25] Tang, Xuanping, Sonja Richter, and Srdjan Nesic. "Study of wettability of different mild steel surfaces." In 17th International Corrosion Congress, vol. 2, p. 1298. 2008.

[26] Quéré, David. "Non-sticking drops." Reports on Progress in Physics 68, no. 11 (2005): 2495.

https://doi.org/10.1088/0034-4885/68/11/R01

[27] Roach, Paul, Neil J. Shirtcliffe, and Michael I. Newton. "Progess in superhydrophobic surface development." Soft matter 4, no. 2 (2008): 224-240.

https://doi.org/10.1039/B712575P

[28] Rashidi, Himan, Zaher Rahimi, and Wojciech Sumelka. "Effects of the slip boundary condition on dynamics and pullin instability of carbon nanotubes conveying fluid." Microfluidics and Nanofluidics 22, no. 11 (2018): 131. https://doi.org/10.1007/s10404-018-2156-z

[29] Choi, Chang-Hwan, K. Johan A. Westin, and Kenneth S. Breuer. "Apparent slip flows in hydrophilic and hydrophobic microchannels." Physics of fluids 15, no. 10 (2003): 2897-2902. https://doi.org/10.1063/1.1605425

[30] Nizkaya, Tatiana V., Evgeny S. Asmolov, Jiajia Zhou, Friederike Schmid, and Olga I. Vinogradova. "Flows and mixing in channels with misaligned superhydrophobic walls." Physical Review E 91, no. 3 (2015): 033020.

https://doi.org/10.1103/PhysRevE.91.033020 